\title{
Osteoporosis is Associated with Varus Deformity in Postmenopausal Women with Knee Osteoarthritis: A Case-Control Study
}

\section{Cheng Zhang}

The First Clinnical Medicle College of Guangzhou University of Chinese Medicine

\section{Zhikun Zhuang}

The First Clinical Medical College of Guangzhou University of Chinese Medicine

\section{Xiaojun Chen}

Guangzhou University of Chinese Medicine

\section{Keda Li}

Liaoning University of Traditional Chinese Medicine

\section{Tianye Lin}

Guangzhou University of Chinese Medicine

\section{Fengxiang Pang}

Guangzhou University of Chinese Medicine

\section{Ying Zhang}

Henan University of Traditional Chinese Medicine

Wei He

Guangzhou University of Traditional Chinese Medicine

Qiushi Wei ( $\nabla$ weiqshi@126.com )

Guangzhou University of Chinese Medicine

\section{Research article}

Keywords: Knee osteoarthritis, Varus deformity, Bone mineral density, Limb alignment Osteoporosis

Posted Date: February 16th, 2021

DOl: https://doi.org/10.21203/rs.3.rs-241591/v1

License: (c) (i) This work is licensed under a Creative Commons Attribution 4.0 International License. Read Full License

Version of Record: A version of this preprint was published at BMC Musculoskeletal Disorders on August 14th, 2021. See the published version at https://doi.org/10.1186/s12891-021-04580-3. 


\section{Abstract}

Background: Varus deformity of the knee is a common pathological characteristic in knee osteoarthritis (KOA), and not enough attention has been given to the relationship between knee varus deformity and the state of systemic bone mass. The purpose of this study is to evaluate the potential relationship between bone mineral density (BMD) and varus deformity in postmenopausal women with KOA.

Methods: This case-control study included 202 postmenopausal women with KOA with or without varus deformity. The hip-knee-ankle angle of the lower extremity (HKA), medial distal femoral angle (MDFA), medial proximal tibial angle (MPTA), and the angle of the joint line (JLCA) were measured in all patients. The BMD of the lumbar (L1-L4), left femoral neck, and left hip were measured by dual-energy X-ray absorptiometry in all patients. The difference in BMD between the knee varus deformity group and the normal limb alignment group was compared, and the relationship between the different angles of limb alignment and the BMD values at different sites was evaluated.

Results: The BMD at different sites of the knee varus deformity group was lower than of the normal limb alignment group, and the incidence of osteoporosis was higher. Pearson correlation analysis showed that BMD of the lumbar spine (L1-L4), left femoral neck and left hip joint were positively correlated with the HKA, but negatively correlated with JLCA. MPTA was positively correlated with the left femoral neck and left hip joint BMD, but not correlated with lumbar bone density. Furthermore, in the normal limb alignment group, the HKA was only negatively correlated with JLCA, but not significantly correlated with MDFA and MPTA. In the varus deformity group, the HKA was not only negatively correlated with JLCA but also positively correlated with MDFA and MPTA.

Conclusions: Osteoporosis should be an important predicting factor for exacerbating knee varus deformity. Maintaining a systemic bone mass level may help to prevent the progression of knee varus deformity in the treatment of KOA.

\section{Introduction}

Knee osteoarthritis (KOA) and osteoporosis are two common diseases in orthopedics. Because the two diseases share common pathogenic factors, such as age, gender, heredity and inflammation, they often coexist in the same patient, especially middle-aged and elderly postmenopausal women [1, 2]. It is worth mentioning that the two diseases are also the main diseases affecting the quality of life of middle-aged and elderly people. Experiencing long-term pain and limited activity lead to a decline in the labor force, which imposes an additional economic burden on society [3].

KOA is no longer considered simple cartilage degeneration but also includes subchondral bone changes, osteophyte formation, synovial inflammation, and changes in ligaments and muscles [4]. Among these effects, increasing attention has been paid to the role of subchondral bone changes in the pathological process of KOA. In previous studies, researchers only focused on the sclerosis of the subchondral plate and the formation of surrounding osteophytes in patients with advanced KOA, suggesting that knee 
osteoarthritis is associated with high bone density. However, in recent years, more studies have found that in the early stage of KOA, subchondral bone resorption increases and bone strength decreases, which in turn cause degeneration of the cartilage above $[5,6]$. Nonetheless, when osteoporosis and KOA coexist in the same patient, there is debate about whether systemic low bone mass exacerbates the progression of KOA.

Varus deformity of the knee joint is not only a key node in the pathological process of knee osteoarthritis but also an important factor for the rapid degeneration of the knee joint. Therefore, the prevention of varus deformity can greatly delay the speed of joint degeneration, which is the key to prevent and treat severe KOA. It is well known that varus deformity may result from congenital anatomical abnormalities, or it may be secondary to medial articular space narrowing after cartilage wear and subluxation of the tibiofemoral joint. However, this cannot fully account for all clinical varus deformity [7]. Although much attention has been given to the effect of periarticular bone mineral density (BMD) on limb alignment [8,9], the effect of systemic BMD, which represents the level of general bone mass, on limb alignment has not been adequately studied.

Full-length, load-bearing anteroposterior computed radiography images of the lower extremities were taken to measure the different angles of limb alignment, such as the hip-knee-ankle angle ( (HKA)), distal femoral medial angle (MDFA), proximal tibial medial angle (MPTA) and the angle of the joint line (JLCA). The purpose of this study was to investigate the relationship between the different angles of the limb alignment and the general bone mass in postmenopausal women with KOA. Our hypothesis was that systemic low bone mass may cause or exacerbate varus deformity in KOA, thereby accelerating the progression of joint degeneration.

\section{Materials And Methods}

We included postmenopausal female patients hospitalized for knee pain in orthopedics at the First Affiliated Hospital of Guangzhou University of Chinese Medicine from January 2018 to June 2020. All of these patients met the American College of Rheumatology diagnostic criteria for KOA [10]. After admission, full-length weighted X-rays of bilateral lower extremities were taken for all patients, and BMD of the lumbar spine (LI-L4), left femoral neck and left hip were measured by dual-energy X-ray absorptiometry. The Kellgren-Lawrence $(\mathrm{KL})$ grade was evaluated by a bilateral lower extremity X-ray. Clinical data, such as age, duration of pain, menstrual history, trauma history, and recent medication use, were collected using an inpatient electronic medical record system. Height and weight were measured prior to the bone density test, and body mass index (BMI) was calculated using height and weight. To reduce the interference of confounding factors in this study, the following exclusion criteria were used: first, patients with knee valgus deformity, as knee valgus is relatively rare clinically and the number of cases is relatively small; second, patients who had taken drugs affecting bone metabolism, such as bisphosphonates and hormones, in the last half year; third, a full-length X-ray of both lower limbs in nonstandard anteroposterior standing position to exclude the measurement error of limb alignment angles caused by rotation of lower extremities; fourth, patients with extra-articular malformations that 
may affect the distribution of the force lines of the knee, such as severe scoliosis, pelvic tilt, hip dysplasia, and angular malformation of the femoral shaft or tibial shaft, etc.; and fifth; patients with pain so severe they cannot walk to reduce the effects of disuse osteoporosis. Finally, 202 postmenopausal women with knee osteoarthritis were enrolled, with an average age of $65.5 \pm 7.4$ years (49-83 years), an average BMI of $26.2 \pm 3.8 \mathrm{~kg} / \mathrm{m} 2$, a median pain duration of 60 months (0.5-360 months), 44 cases of grade KL2, 79 cases of grade KL3, and 79 cases of grade KL4.

Measurement of different angles of limb alignment

After admission, full-length weight-bearing X-ray films of both lower limbs were taken, and four different angles of HKA, MDFA, MPTA and JLCA that could reflect the limb alignment were measured. If patients had been diagnosed with bilateral KOA, the more severe side was

measured. The mechanical axis of the femur was defined as the connection between the center of the femoral head and the center of the femoral condyle, while the mechanical axis of the tibia was defined as the connection between the center of the tibial plateau and the center of the ankle joint. The degree of knee varus was defined by the HKA, i.e., the medial intersection angle between the mechanical axis of the femur and the mechanical axis of the tibia. The smaller the HKA was, the more serious the varus deformity was. According to the standard range of the physiological HKA of Chinese women [11], we defined $175.3^{\circ} \leq \mathrm{HKA} \leq 180.3^{\circ}$ as normal limb alignment and an HKA $<175.3^{\circ}$ as varus deformity. The other three angles were defined as follows: MDFA as the medial angle between the mechanical shaft of the femur and the tangential line of the femoral condyle, MPTA as the medial angle between the mechanical shaft of the tibia and the tangential line of the tibial plateau, and JLCA as the lateral angle between the tibial plateau tangent and the femoral condyle tangent (Fig. 1).

To reduce measurement errors, the same researcher (the first author) took all measurements using the same imaging system successively at two-week intervals. The investigator was unaware of the patient's bone mineral density and other clinical data prior to measurement.

Measurement of bone density

After admission, BMD was measured at the left femoral neck, left hip and lumbar spine (L1-L4) by dual energy X-ray absorptiometry (GE Lunar Prodigy, USA). The BMD of these three regions can sufficiently reflect the bone mass of the whole body and is the gold standard for the diagnosis of osteoporosis. According to WHO diagnostic criteria, $\mathrm{T} \leq-2.5$ is diagnosed as osteoporosis, $-2.5<\mathrm{T}<-1$ is diagnosed as osteopenia, and $\geq-1$ is normal bone density. Measurement parameters and machine quality are regularly checked according to the instructions provided by the manufacturer.

\section{Statistical analysis}

After the normal distribution test of the numerical variables, the Student 's t-test was used for the intergroup comparison of age, BMI and BMD values at different sites. Conversely, the Mann-Whitney U 
test was used for the intergroup comparison of the duration of pain. The

chi-square test was used to compare the prevalence of osteoporosis. Binary logistic regression analysis was used to determine the risk factors affecting varus deformity. The Pearson correlation coefficient was used to evaluate the correlation between BMD at different positions and different angles of limb alignment. Similar to age, BMI and duration of pain may affect the HKA. Multiple linear regression was used to evaluate the influence of BMD values at different sites on the HKA after adjusting for confounding factors, such as age, BMI and duration of pain. Finally, the Pearson correlation coefficient was used to evaluate the correlation between the HKA and other angles in the varus group and the normal limb alignment group.

All data analyses were performed with IBM SPSS 24.0 statistical software, and a $P$-value less than 0.05 was considered statistically significant in all tests.

\section{Results}

In the varus deformity group (HKA $\left.<175.3^{\circ}\right)$, there were 144 cases, including 69 cases of osteoporosis, 60 cases of osteopenia and 15 cases of normal bone mass. There were 58 cases in the normal limb alignment group $\left(175.3^{\circ} \leq \mathrm{HKA} \leq 180.3^{\circ}\right)$, including 15 cases of osteoporosis, 34 cases of osteopenia, and 9 cases of normal bone mass. There were no statistically significant differences in age, BMI and pain duration between the two groups. The BMD of the lumbar spine (L1-L4), left femoral neck and left hip of the varus deformity group was significantly lower than that of the normal limb alignment group, and the incidence of osteoporosis (47.9\%) was significantly higher than that of the normal limb alignment group $(25.9 \%, P<0.05)($ Table 1$)$. 
Table 1

Comparison of characteristics, bone mineral density and prevalence of osteoporosis

\begin{tabular}{|c|c|c|c|}
\hline & Varus group & Normal alignment group & $p$-value \\
\hline Numbers & 144 & 58 & \\
\hline Age (years) & $66.1 \pm 7.6$ & $64.0 \pm 6.7$ & 0.068 \\
\hline $\mathrm{BMI}\left(\mathrm{kg} / \mathrm{m}^{2}\right)$ & $26.1 \pm 3.9$ & $26.3 \pm 3.3$ & 0.808 \\
\hline Pain duration (month) & $60(0.5-360)$ & $36(1.0-240.0)$ & 0.355 \\
\hline \multicolumn{4}{|l|}{$\mathrm{BMD}\left(\mathrm{g} / \mathrm{cm}^{2}\right)$} \\
\hline L1-L4 Spine & $0.84 \pm 0.15$ & $0.94 \pm 0.17$ & $<0.001$ \\
\hline Femoral neck & $0.67 \pm 0.13$ & $0.74 \pm 0.13$ & 0.002 \\
\hline Total hip & $0.78 \pm 0.14$ & $0.85 \pm 0.13$ & 0.001 \\
\hline \multicolumn{3}{|l|}{ Prevalence } & 0.006 \\
\hline Osteoporosis (N,\%) & $69(47.9)$ & $15(25.9)$ & \\
\hline Osteopenia $(\mathrm{N}, \%)$ & $60(41.7)$ & $34(58.6)$ & \\
\hline Normal BMD (N,\%) & $15(10.4)$ & $9(15.5)$ & \\
\hline \multicolumn{4}{|c|}{ Data are presented as the mean \pm SD for age, $\mathrm{BMI}$, and median (range) for pain duration } \\
\hline \multicolumn{4}{|c|}{ BMI: body mass index, BMD: bone mineral density } \\
\hline $\begin{array}{l}\text { Binary logistic regressic } \\
\text { knee varus deformity. A } \\
\text { adjusted OR value was }\end{array}$ & $\begin{array}{l}\mathrm{d} \text { that osteopor } \\
\text { sting for confab } \\
5 \% \text { Cl: } 1.215-5 .\end{array}$ & $\begin{array}{l}\text { uld significantly influence } \\
\text { factors, such as age, BMI } \\
<0.05 \text { ) (Table 2). }\end{array}$ & $\begin{array}{l}\text { rence of } \\
\text { time, the }\end{array}$ \\
\hline
\end{tabular}

Table 2

Adjusted analysis performed with a binary logistic regression model for knee varus deformity

\begin{tabular}{|lllll|}
\hline & $\boldsymbol{\beta}$ & Adjusted OR & 95\% Cl for Adjusted OR & $\boldsymbol{p}$-value \\
\hline Age (years) & 0.020 & 1.020 & $0.974-1.068$ & 0.394 \\
\hline BMl $\left(\mathrm{kg} / \mathrm{m}^{2}\right)$ & 0.013 & 1.013 & $0.930-1.103$ & 0.774 \\
\hline Pain duration (month) & 0.001 & 1.001 & $0.996-1.007$ & 0.625 \\
\hline Osteoporosis & 0.915 & 2.498 & $1.215-5.134$ & 0.013 \\
\hline BMl: body mass index, OR: odds ratio, Cl: confidence interval & \\
\hline
\end{tabular}


According to Pearson correlation analysis, among all participants, the BMD of the lumbar spine (L1 - L4), the left femoral neck and left hip was positively correlated with the HKA, and negatively correlated with JLCA. MPTA was positively correlated with BMD values of the left femoral neck and the left hip joint, but not with those of the lumbar spine. MDFA was not correlated with BMD values of the above three sites (Fig. 2).

BMD: bone mineral density, HKA: hip-knee-ankle angle, MDFA: distal femoral medial angle, MPTA: proximal tibial medial angle, JLCA: angle of the joint line

In the multiple linear regression model, after adjusting for confounding factors, such as age, BMI, and pain duration, the BMD of the lumbar spine (L1-L4), the left femoral neck, and the left hip could have a significant positive influence on the value of the HKA (Table 3). That is, with increasing BMD, the degree of varus deformity would be reduced.

Table 3

The relationship between BMD at different sites and HKA after adjusting for confounding factors

\begin{tabular}{|lccllll|}
\hline & B & S.E. & Standardized $\boldsymbol{\beta}$ & 95\% Cl for B & p-value \\
\hline L1-L4 BMD & 4.904 & 2.330 & 0.154 & $0.310-9.498$ & 0.037 \\
\hline Femoral neck BMD & 7.597 & 3.024 & 0.199 & $1.634-13.560$ & 0.013 \\
\hline Total hip BMD & 3.024 & 3.024 & 0.208 & $1.904-13.829$ & 0.010 \\
\hline HKA: hip-knee-ankle angle, BMD: bone mineral density, Cl: confidence interval \\
\hline
\end{tabular}

Finally, in the normal limb alignment group, Pearson correlation analysis showed that the HKA was only negatively correlated with JLCA but had no significant correlation with MDFA and MPTA, whereas in the varus deformity group, HKA was not only negatively correlated with JLCA but also positively correlated with MDFA and MPTA (Fig. 3).

\section{Discussion}

Studies on the relationship between osteoporosis and osteoarthritis are ongoing. In early studies, high bone mineral density was generally believed to be related to the incidence and progression of osteoarthritis. Although recent studies began to realize that osteoporosis plays an important role in the pathological process of KOA, the relationship between osteoporosis and KOA is still controversial. Part of the reason may be that patients in different studies were at different stages of disease and had different indicators for measuring the progression of KOA [12]. KL grade pays more attention to the formation of osteophyte and the narrowing of joint space [13], while limb alignment can more directly reflect the incidence of varus deformity, which is key to the rapid progression of KOA. 
Previous studies have primarily focused on the relationship between the subchondral BMD and limb alignment. Yoshinori et al. found that in patients with medial compartment KOA, the medial condyles had significantly higher BMD than the lateral condyles in both the femur and tibia, and the medial-to-lateral BMD ratio was negatively correlated with the HKA and the inclination angle of the tibial plateau [14]. Similarly, Grace H. Lo reported that in patients with KOA, the severity of knee varus deformity was positively correlated with the subchondral BMD of the medial tibia platform [15]. However, these studies can only reflect the results of the change in periarticular BMD caused by the uneven change in mechanical stress, and it is difficult to explain the influence of BMD on the change in limb alignment. Moreover, local BMD could not represent the general bone mass, and it was difficult to reveal the relationship between the general bone mass state and limb alignment.

In this study, we measured the BMD at the lumbar spine, hip joint and femoral neck to represent the state of general bone mass and measured the different angles of limb alignment to evaluate the relationship between knee varus deformity and systemic BMD. We found that the BMD values of the above three sites in varus deformity patients were significantly lower than those in the normal limb alignment group, and the incidence of osteoporosis was higher than that in the normal limb alignment group. Furthermore, after adjusting for confounding factors, such as age, BMI, and pain duration, regression analysis showed that the BMD at the lumbar spine (L1-L4), the left femoral neck, and the left hip could have a significant positive influence on the value of the HKA, suggesting that a generalized low bone mass status may influence the severity of varus deformity in patients with KOA. Consistent with the results of this study, Gun-IL Im et al. also reported that proximal femur BMD was negatively correlated with $\mathrm{KL}$ grading and positively correlated with medial tibiofemoral joint space and the HKA [16].

In recent years, substantial evidence has shown that subchondral bone and cartilage are a closely related integrated unit and mutually adaptable [17-19]. In the early stage of KOA, the absorption of subchondral bone is enhanced, and the mechanical strength of the bone is reduced. Improper stress stimulation also induces subchondral microfracture, which causes bone marrow edema and then degeneration of the cartilage covered on the surface $[20,21]$. Y. Chen's study on tibial plateau specimens of patients with knee osteoarthritis found that subchondral bone cysts degeneration was caused by increased local bone remolding, and the structural change in the cysts region accelerated the destruction of the overlying cartilage [22]. Zhu et al. even reported that the source of pain of KOA might be related to the abnormal activity of subchondral osteoclasts, which secreted Netrin-1 and induced neuronal axonal growth [23]. Therefore, we speculated that if patients with KOA also have osteoporosis, their subchondral mechanical properties will be further reduced, which will accelerate the progress of knee osteoarthritis and even varus deformity.

MDFA reflects the bone morphology of the medial femoral condyle, while MPTA reflects the inclination of the medial tibial plateau and JLCA is related to the narrowing degree of the medial articular space. Interestingly, in our study, BMD at the lumbar spine, femoral neck and hip joint is not only significantly correlated with the HKA, but significantly positively correlated with MTPA and negatively correlated with JLCA, indicating that with decreasing BMD, the more severe the inclination of the medial tibial plateau 
and the narrower the medial articular space would be. In addition, in the normal limb alignment group, the HKA was only negatively correlated with JLCA but not significantly correlated with MDFA and MPTA. In contrast, HKA was not only negatively correlated with JLCA but also positively correlated with MDFA and MPTA angles in the varus deformity group. The above results indicate that varus deformity of the knee is not only related to the narrowing of the medial articular space but may also be related to the change in the bone structure around the joint.

In fact, subchondral bone provides structural support for articular cartilage and plays a role in shock absorption, and its mechanical structure changes are closely related to cartilage degeneration [24]. Considering the increased fragility of the subchondral bone in osteoporosis patients, subchondral bone remolding will be further enhanced in the early stage of KOA [25]. At the same time, the stress of the medial platform is higher than that of the lateral platform under physiological conditions [26]. Therefore, we hypothesized that when KOA patients have osteoporosis, due to the weakening of the subchondral bone strength and improper stress stimulation, the medial platform may subside, thus aggravating the existing varus deformity caused by cartilage wear. In this study, MTPA was positively correlated with BMD values of the three different sites, which can confirm our inference to a certain extent. With the progression of knee varus, the stress in the medial compartment will further increase, which will aggravate the cartilage damage, thus forming a vicious circle. One study also reported that osteoporosis causes attrition of bone structure in advanced KOA, aggravating the deformity of joints [27]. Therefore, these results suggest that maintaining the systemic bone mass of patients may effectively delay the progression of KOA, especially in patients with osteoporosis.

There are several limitations in this study. First, this is a cross-sectional, retrospective study design in which we were unable to longitudinally observe the dynamic changes in the influence of osteoporosis on varus deformity. Second, as a deficiency of estrogen is a common cause of osteoporosis and KOA, an osteoporosis clinical merger with KOA is often seen in postmenopausal women, so we only included postmenopausal Chinese women. To extend the conclusion to men and other races, research with a large sample of other populations should be conducted to confirm our findings. Third, we failed to observe the changes of BMD in the subchondral bone, as the BMD around the knee joint is not the regular detection area of BMD in our hospital. Therefore, it is necessary to further observe the correlation between periarticular BMD and systemic BMD in patients with KOA in future studies. Despite these limitations, this study still revealed the relationship between the systemic bone mass status and different angles of limb alignment and proposed that osteoporosis may be an independent risk factor for varus deformity. Our study results will also provide a reference to further reveal the internal relationship between osteoporosis and knee osteoarthritis.

\section{Conclusion}

In summary, the incidence of knee varus deformity is not only related to the narrowing of the joint space but also to the change in the bone structure around the joint, which may be an important factor. 
Maintaining systemic bone mass may help prevent varus deformity from progressing, but more longitudinal studies are needed to confirm this conclusion.

\section{Abbreviations}

KOA: Knee osteoarthritis; BMD: Bone mineral density; HKA: Hip-knee-ankle angle; MDFA: Medial distal femoral angle; MPTA: Medial proximal tibial angle; JLCA: Angle of the joint line; KL: Kellgren-Lawrence score; BMI: Body mass index; WHO: World Health Organization

\section{Declarations}

\section{Acknowledgments}

Not Applicable

\section{Author contributions}

QS W and WH designed the study. CZ and ZK Z performed the statistical analysis and drafted the manuscript. XJ C, FX P and TY $L$ were responsible for data collection and outcomes evaluation. $K D L$ and $Y Z$ contributed to measurement of radiographic data. QS W contributed to revision of the manuscript. All authors read and approved the final manuscript.

\section{Funding}

This study was funded by Natural Science Foundation of China (81873327), Natural Science Foundation of Guangdong (2015A030313353), Excellent Doctoral Dissertation Incubation Grant of First Clinical School of Guangzhou University of Chinese Medicine.

\section{Availability of data and materials}

The datasets used and/or analysed during the current study are available from the corresponding author on reasonable request.

\section{Ethics approval and consent to participate}

Retrospective observational studies were conducted based on the data obtained for clinical purposes in this study. We consulted the Ethics Committee of the First Affiliated Hospital of Guangzhou University of Chinese Medicine, and they determined that our study did not need ethical approval. 


\section{Consent for publication}

Not applicable

\section{Competing interests}

The authors declare that they have no competing financial interests

\section{Author details:}

${ }^{1}$ The First Clinical Medical College of Guangzhou University of Chinese Medicine, Guangzhou 510405, Guangdong, China. ${ }^{2}$ Department of Joint Orthopaedic, the Third Affiliated Hospital, Guangzhou University of Chinese Medicine, Guangzhou, 510405, Guangdong, China. ${ }^{3}$ Institude of Orthopedica, Guangzhou University of Chinese Medicine, Guangzhou 510405,Guangdong, China. ${ }^{4}$ Quanzhou Osteopathic Hospital Affiliated to Fujian University of Traditional Chinese Medicine, Quanzhou 362000, Fujian, China. ${ }^{5}$ Liaoning University of Chinese Medicine, Shengyang 110033, Liaoning, China. ${ }^{6}$ Henan provincial Orthopedic Hospital, Luoyang 471000, Henan, China.

\section{References}

1. Geusens PP, van den Bergh JP. Osteoporosis and osteoarthritis: shared mechanisms and epidemiology. Curr Opin Rheumatol. 2016;28(2):97-103.

2. Bultink IE, Lems WF. Osteoarthritis and osteoporosis: what is the overlap? Curr Rheumatol Rep. 2013;15(5):328.

3. Tang X, Wang S, Zhan S, Niu J, Tao K, Zhang Y, et al. The Prevalence of Symptomatic Knee Osteoarthritis in China: Results From the China Health and Retirement Longitudinal Study. Arthritis \& rheumatology (Hoboken, NJ) 2016;68 (3):648-653.

4. Kolasinski SL, Neogi T, Hochberg MC, Oatis C, Guyatt G, Block J, et al. 2019 American College of Rheumatology/Arthritis Foundation Guideline for the Management of Osteoarthritis of the Hand, Hip, and Knee. Arthritis Care Res. 2020;72(2):149-62.

5. Chu L, Liu X, He Z, Han X, Yan M, Qu X, Li X, Yu Z. Articular Cartilage Degradation and Aberrant Subchondral Bone Remodeling in Patients with Osteoarthritis and Osteoporosis. J Bone Miner Res. 2020;35(3):505-15.

6. Barr AJ, Campbell TM, Hopkinson D, Kingsbury SR, Bowes MA. Conaghan PG.A systematic review of the relationship between subchondral bone features, pain and structural pathology in peripheral joint osteoarthritis. Arthritis Res Ther. 2015;17:228.

7. Khamaisy S, Nam D, Thein R, Rivkin G, Liebergall M, Pearle A. Limb alignment, subluxation, and bone density relationship in the osteoarthritic varus knee. J Knee Surg. 2015;28(3):207-12. 
8. Akamatsu Y, Mitsugi N, Taki N, Kobayashi H, Saito T. Medial versus lateral condyle bone mineral density ratios in a cross-sectional study: a potential marker for medial knee osteoarthritis severity. Arthritis Care Res. 2012;64(7):1036-45.

9. Neogi T, Nevitt M, Niu J, Sharma L, Roemer F, Guermazi A, et al. Subchondral bone attrition may be a reflection of compartment-specific mechanical load: the MOST Study. Annals of the rheumatic diseases.2010; 69 (5):841-844.

10. Damen J, van Rijn RM, Emans PJ, Hilberdink W, Wesseling J, Oei EHG, et al. Prevalence and development of hip and knee osteoarthritis according to American College of Rheumatology criteria in the CHECK cohort. Arthritis Res Ther. 2019;21(1):4.

11. Tang WM, Zhu YH. ,Chiu KY.Axial Alignment of the Lower Extremity in Chinese Adults. J Bone Joint Surg Am. 2000;82(11):1603-8.

12. Castaneda S, Roman-Blas JA, Largo R, Herrero-Beaumont G. Osteoarthritis: a progressive disease with changing phenotypes. Rheumatology. 2014;53(1):1-3.

13. Kim YH, Lee JS, Park JH. Association between bone mineral density and knee osteoarthritis in Koreans: the Fourth and Fifth Korea National Health and Nutrition Examination Surveys. Osteoarthritis Cartilage. 2018;26(11):1511-7.

14. Ishii Y, Noguchi H, Sato J, Ishii H, Todoroki K, Toyabe SI. Association between bone mineral density distribution and various radiographic parameters in patients with advanced medial osteoarthritis of the knee. J Orthop Sci. 2019;24(4):686-92.

15. Lo GH, Merchant MG, Driban JB, Duryea J, Price LL, Eaton CB, et al. Knee Alignment Is Quantitatively Related to Periarticular Bone Morphometry and Density, Especially in Patients With Osteoarthritis. Arthritis \& rheumatology (Hoboken, NJ) 2018;70 (2):212-221.

16. Im GI, Kwon OJ, Kim CH. The relationship between osteoarthritis of the knee and bone mineral density of proximal femur: a cross-sectional study from a Korean population in women. Clin Orthop Surg. 2014;6(4):420-5.

17. Omoumi P, Babel H, Jolles BM, Favre J. Relationships between cartilage thickness and subchondral bone mineral density in non-osteoarthritic and severely osteoarthritic knees: In vivo concomitant 3D analysis using CT arthrography. Osteoarthritis Cartilage. 2019;27(4):621-9.

18. Omoumi P, Babel H, Jolles BM, Favre J. Quantitative regional and sub-regional analysis of femoral and tibial subchondral bone mineral density (SBMD) using computed tomography (CT): comparison of non-osteoarthritic (OA) and severe OA knees. Osteoarthritis Cartilage. 2017;25(11):1850-7.

19. Burnett WD, Kontulainen SA, McLennan CE, Hazel D, Talmo C, Wilson DR, et al. Knee osteoarthritis patients with more subchondral cysts have altered tibial subchondral bone mineral density. BMC Musculoskelet Disord. 2019;20(1):14.

20. Ota S, Chiba D, Sasaki E, Kumagai G, Yamamoto Y, Nakaji S, et al. Symptomatic bone marrow lesions induced by reduced bone mineral density in middle-aged women: a cross-sectional Japanese population study. Arthritis Res Ther. 2019;21(1):113.

21. Suri S, Walsh DA. Osteochondral alterations in osteoarthritis. Bone. 2012;51(2):204-11. 
22. Chen $\mathrm{Y}$, Wang T, Guan M, Zhao W, Leung FK, Pan H, et al. Bone turnover and articular cartilage differences localized to subchondral cysts in knees with advanced osteoarthritis. Osteoarthritis Cartilage. 2015;23(12):2174-83.

23. Zhu S, Zhu J, Zhen G, Hu Y, An S, Li Y, et al. Subchondral bone osteoclasts induce sensory innervation and osteoarthritis pain. J Clin Investig. 2019;129(3):1076-93.

24. Lo GH, Schneider E, Driban JB, Price LL, Hunter DJ, Eaton CB, et al. Periarticular bone predicts knee osteoarthritis progression: Data from the Osteoarthritis Initiative. Semin Arthritis Rheum. 2018;48(2):155-61.

25. Herrero-Beaumont G, Roman-Blas JA. Osteoarthritis. Osteoporotic OA: a reasonable target for boneacting agents. Nat Rev Rheumatol. 2013;9(8):448-50.

26. Herrero-Beaumont G, Roman-Blas JA, Bruyere O, Cooper C, Kanis J, Maggi S, et al. Clinical settings in knee osteoarthritis: Pathophysiology guides treatment. Maturitas. 2017;96:54-7.

27. Linde KN, Puhakka KB, Langdahl BL, Soballe K, Krog-Mikkelsen I, Madsen F, et al. Bone Mineral Density is Lower in Patients with Severe Knee Osteoarthritis and Attrition. Calcif Tissue Int. 2017;101(6):593-601.

\section{Figures}



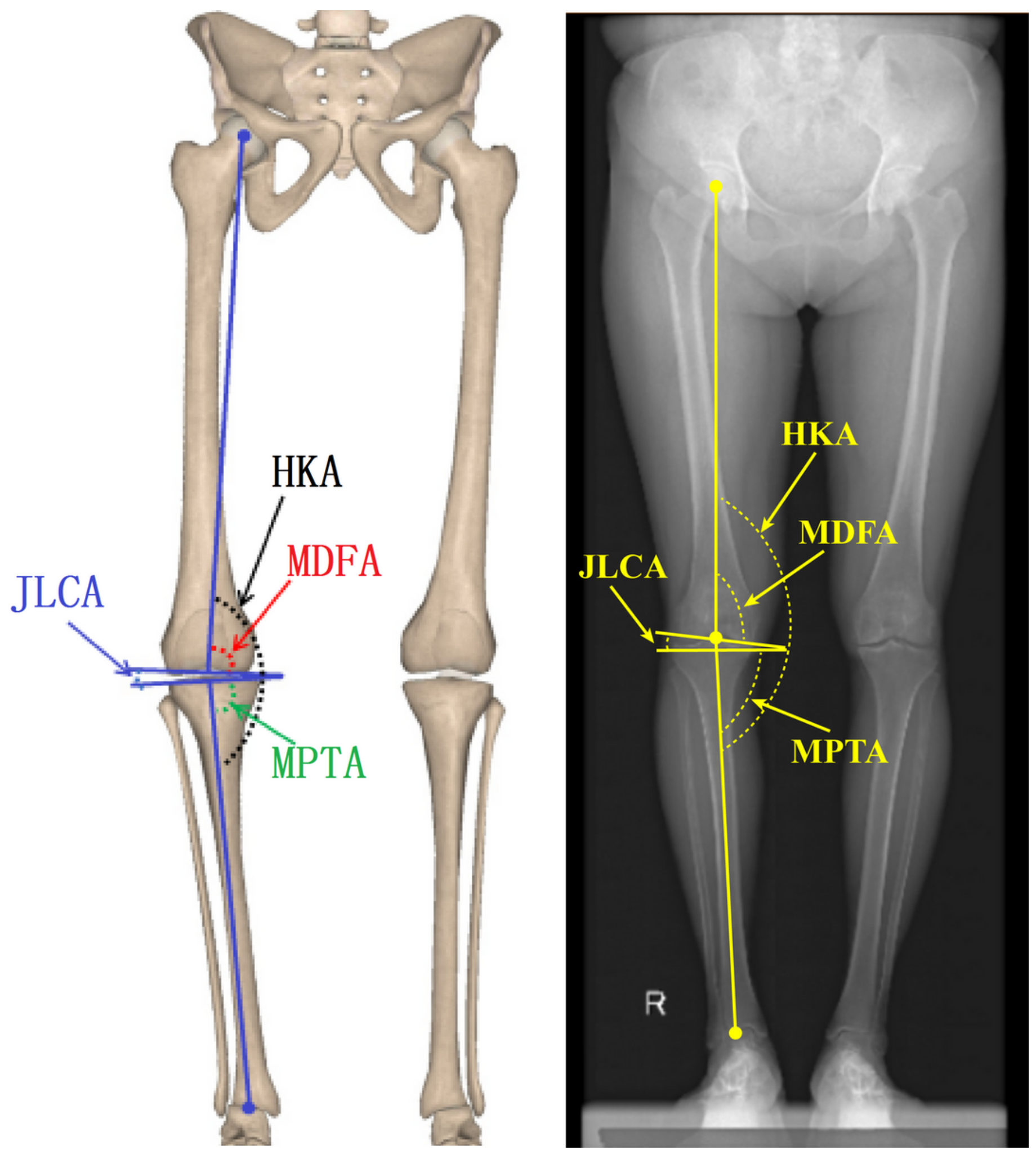

\section{Figure 1}

Schematic diagram of the mechanical angle of lower extremity (HKA), distal medial femoral angle (MDFA), proximal medial tibial angle (MPTA) and angle of joint line (JLCA). 

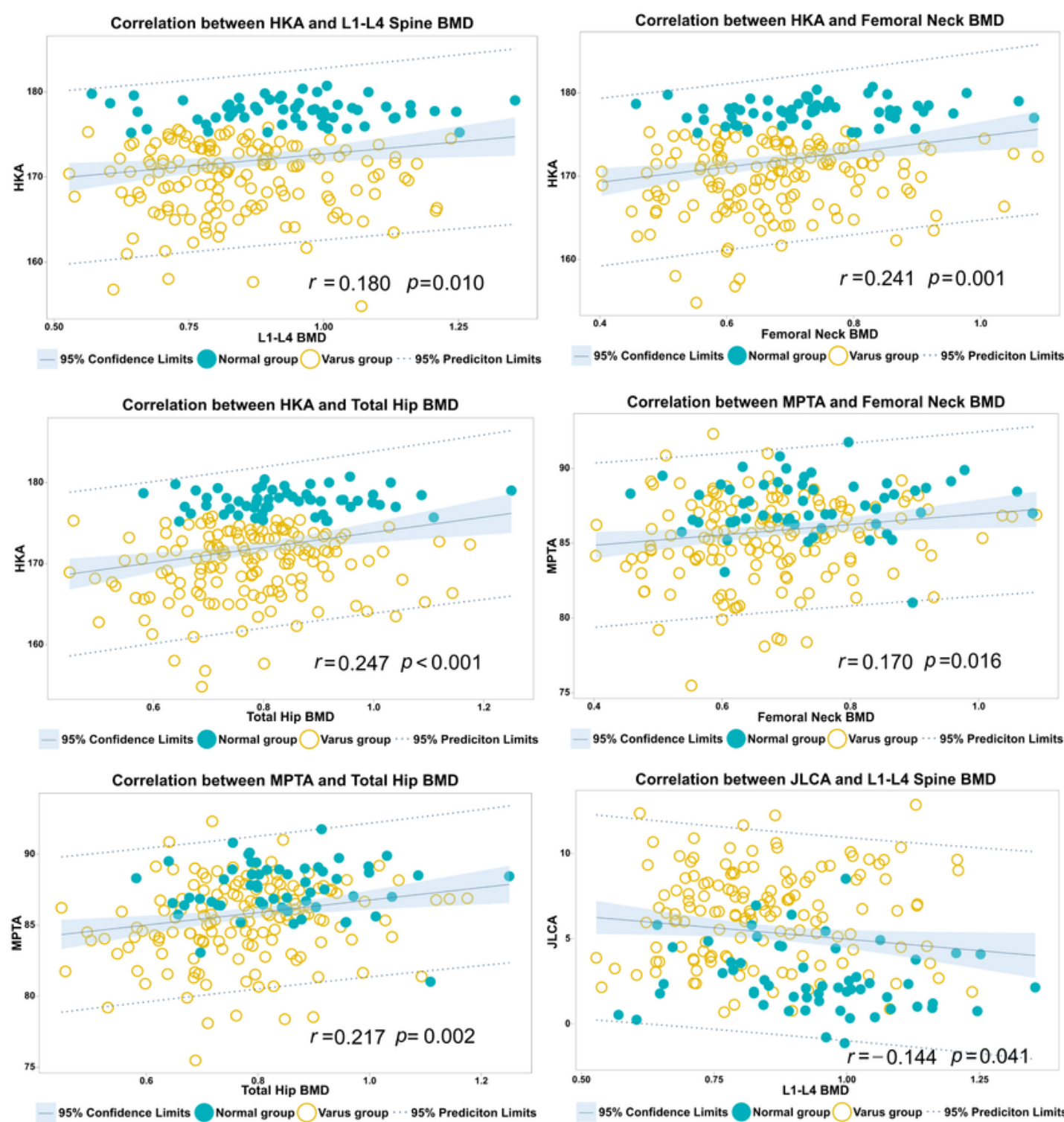

- $95 \%$ Confidence Limits $\bigcirc$ Normal group $\bigcirc$ Varus group $\cdots 95 \%$ Prediciton Limits
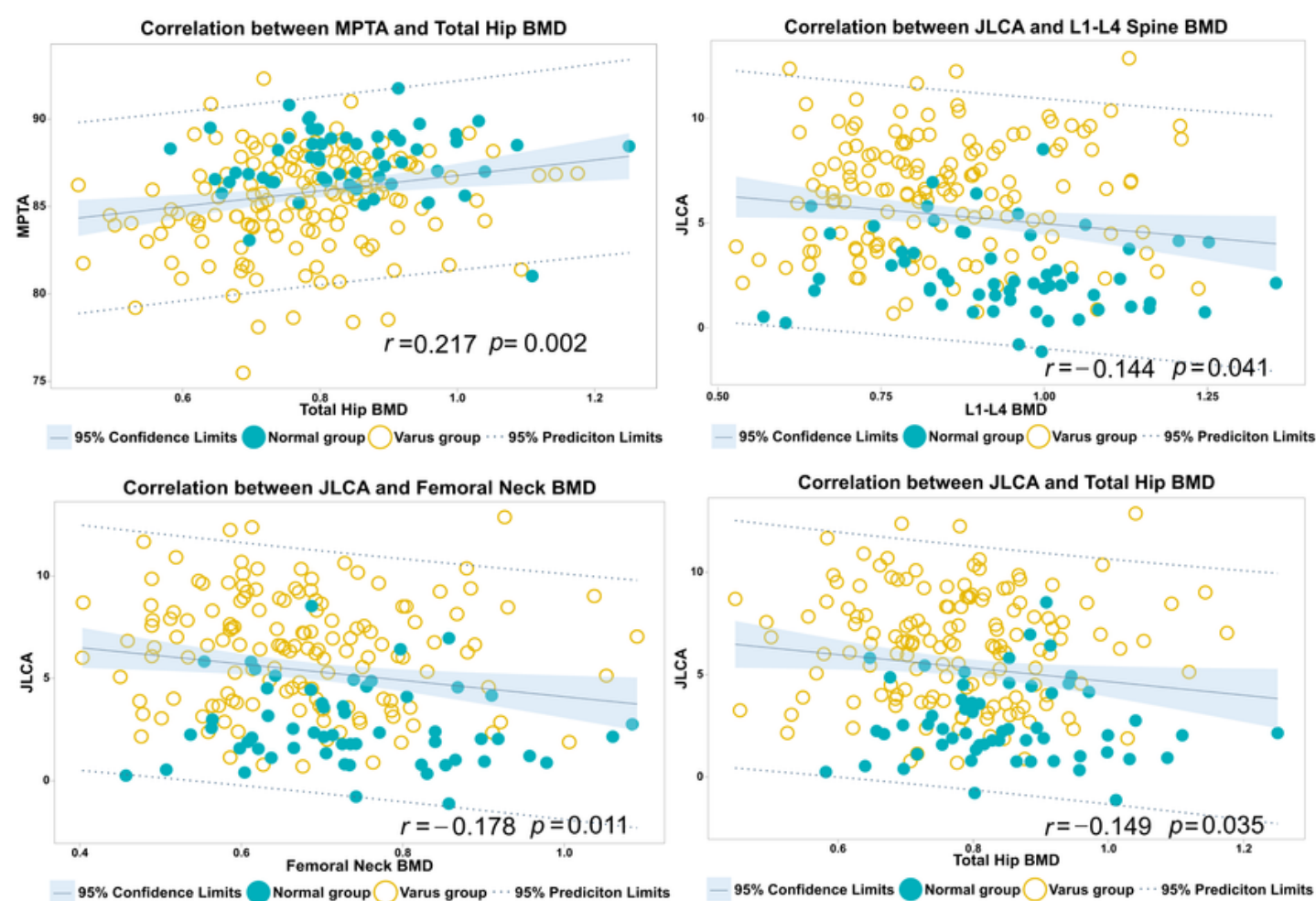

\section{Figure 2}

Correlation between BMD values of different sites and different angles of limb alignment BMD: bone mineral density, HKA: hip-knee-ankle angle, MDFA: distal femoral medial angle, MPTA: proximal tibial medial angle, JLCA: angle of the joint line 

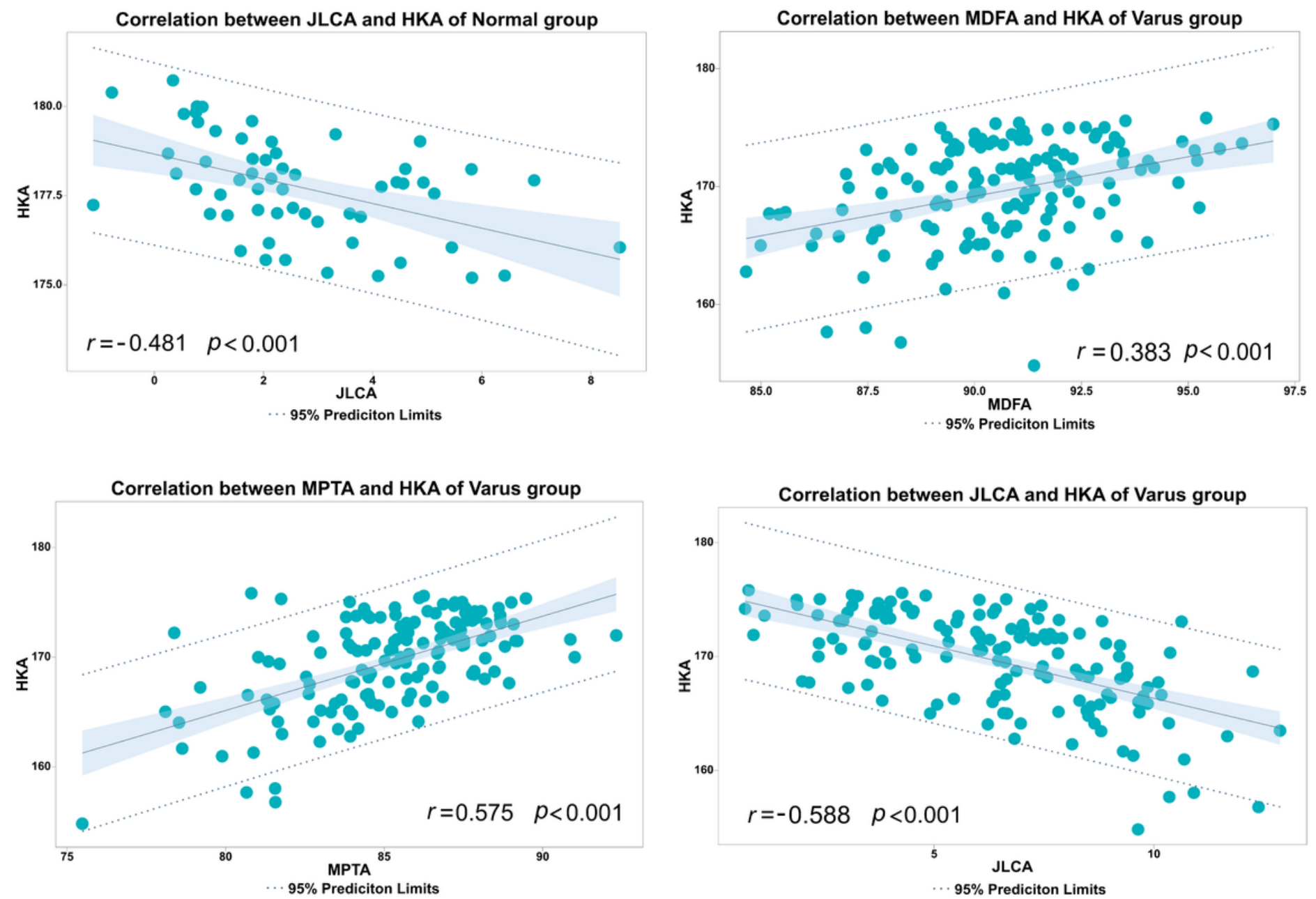

\section{Figure 3}

Correlation analysis of the HKA with MDFA, MPTA and JLCA 